УДК $541.1+628.16$

\title{
Математическое моделирование и расчет процесса деминерализации воды в промышленном фильтре Сообщение 2. Влияние изменения производительности и состава исходной воды на количество и качество очищенной воды
}

\author{
Комарова И.В., Галкина Н.К., Прудковский А.Г. \\ Институт геохимии и аналитической химии им. В.И.Вернадского РАН, Москва
}

Поступила в редакцию 25.12.2016 г.

\begin{abstract}
В данном сообщении рассмотрено влияние изменения состава исходной очищаемой воды и производительности (скорости потока) на процесс очистки воды на полностью регенерированном ионите, для чего в промышленный фильтр был загружен новый катионит КУ-2 в водородной форме. Получены выходные кривые иона натрия на выходе из фильтра и из середины слоя катионита. Расчетная часть работы выполнена по программе «Createscheme», позволяющей учитывать переменный характер входных параметров. Сопоставление экспериментальных выходных кривых с расчетными показало, что наилучшее их совпадение наблюдается при учете всех изменений состава воды на входе в фильтр и скорости потока по сравнению с моделью с использованием постоянных граничных условий.
\end{abstract}

Ключевые слова: состав очищаемой воды, производительность фильтра, изменение входных параметров, расчетные и экспериментальные выходные кривые.

\section{Mathematical simulation and calculation of the process of water demineralization in industrial filter Part 2. Influence of filter productivity and water composition changing on quality and quantity of purified water}

\author{
Komarova I.V., Galkina N.K., Prudkovskii A.G. \\ Vernadsky Institute of Geochemistry and Analytical Chemistry of Russian Academy of Sciences. Moscow
}

The aim of this work was to study the effect of changing the composition of raw water feeding the cationic filter and the flow rate on the performance of water treatment process for power plant.

Experiments were carried out at the Novocherkassk Power Plant central desalting unit, on an industrial filter loaded with the fresh cationic resin KU-2 in hydrogen form. Sodium concentration histories were measured at the output and in the middle bed point of the filter and dynamic break-through curves were plotted. The impact of changes in the composition of feeding water and its flow rate on the purification process was estimated by the comparison of experimental and calculated break-through. Two types of computation programs were compared, and the first one was for calculating single-component dynamics for film diffusion at sorption, with using mean values of the component concentrations. Another one was the «Createscheme» program, which allowed taking into account changes in boundary data. In the last case, best agreement between experimental and calculated data were shown, indicating the adequacy of the developed mathematical model to the real industrial process characterized with a variety of technological features. 
Key words: composition of water under treatment, flow rate of filter, changing the input data, calculated and experimental break-through curves

\section{Введение}

Настоящий цикл работ посвящен изложению результатов экспериментов на промышленном ионообменном фильтре и соответствующих расчетов динамики массопереноса на различных стадиях процесса водоподготовки с использованием новой программы «Createscheme», построенной на математической модели, позволяющей учитывать переменные граничные условия и описанной в предыдущем сообщении. Ранее, еще до создания предложенного здесь более общего подхода, нами проводились расчеты времени до проскока натрия на основе модели динамики ионного обмена в однокомпонентной системе при внешней диффузии с постоянными значениями скорости потока и исходного солесодержания [1]. Расчетные и экспериментальные данные при средней скорости потока и среднем суммарном содержании ионов в очищаемой осветленной воде подтвердило адекватность математической модели процессу очистки воды независимо от масштаба фильтра и свойств сорбента.

Для расчета очистки воды с переменными исходными данными может быть использована только программа расчета многокомпонентной динамики сорбции. Это связано с тем, что при изменении исходных данных для продолжения расчета необходимо знание распределения всех сорбированных компонентов по слою ионита, что невозможно получить по модели для расчета однокомпонентной динамики сорбции.

\section{Эксперимент}

Особенность описанного в данной работе промышленного эксперимента заключается в том, что процесс очистки воды осуществлялся на катионите КУ-2, полностью (100\%) регенерированном и отмытом частично обессоленной водой, полученной на соседних работающих фильтрах первой ступени. В течение рабочего цикла природная вода после предварительной очистки подавалась на исследуемый фильтр сверху вниз через задвижки 1 и 2, как показано на рис.1 первого сообщения настоящего цикла сообщений. Исследуемый фильтр был включен в общую технологическую схему обессоливания воды, поэтому режим работы фильтра должен был определяться технологическим регламентом.

Во время эксперимента фильтр был отключен на регенерацию по проскоку

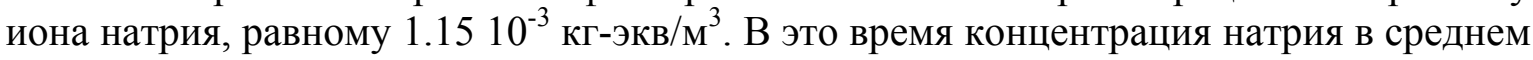
сечении фильтра достигает значения, равного общему солесодержанию очищаемой воды.

На рис.1 представлены экспериментальная выходная кривая натрия в середине фильтра в сравнении с расчетными выходными кривыми при средней скорости потока и среднем солесодержании, полученными по программе однокомпонентной динамики сорбции для внешней диффузии и по программе «Createscheme» [2]. Эти данные свидетельствуют, что математическая модель, описывающая стадию сорбции на катионите в водородной форме с параметрами, полученными в лабораторных условиях (табл.1 сообщение 1), применима к промышленному фильтру.

В процессе рабочего цикла состав очищаемой воды изменялся несколько раз в связи с периодическими изменениями режимов предварительной очистки природной воды. Переключения других параллельно работающих фильтров общей схемы во время проводимого нами эксперимента приводило к изменению производительности исследуемого фильтра.

Комарова и др. / Сорбционные и хроматографические процессы. 2017. Т. 17. № 1 


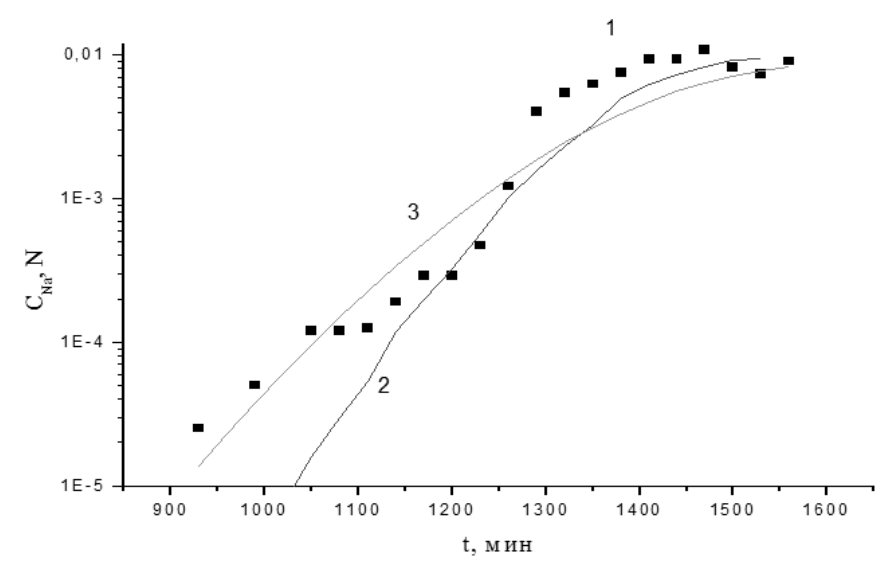

Рис. 1. Экспериментальная выходная кривая натрия в среднем сечении фильтра (1) в сравнении с выходными кривыми, рассчитанными по программам:

2 -однокомпонентная динамика ионного обмена при внешней диффузии 3 программа «Createscheme» $\mathrm{C}_{0}=0.01 \mathrm{~N}, \mathrm{~V}=65 \mathrm{~m}^{3} /$ час

В таблице 1 представлены данные по составу исходной воды и производительности фильтра в течение рассматриваемого эксперимента.

Таблица 1. Этапы изменения входных параметров в течение рабочего цикла

\begin{tabular}{|c|c|c|c|c|c|c|c|}
\hline № & Время, мин & $\begin{array}{c}\mathrm{Na}, \\
\text { моль/дм }\end{array}$ & $\begin{array}{c}\mathrm{Mg} \\
\text { моль/дм } \\
\end{array}$ & $\begin{array}{c}\mathrm{Ca} \\
\text { моль/дм }\end{array}$ & $\begin{array}{c}\mathrm{Cl} \\
\text { моль/дм } \\
\end{array}$ & $\begin{array}{c}\text { Vcp, } \\
\mathrm{m}^{3} / \mathrm{ч} a c\end{array}$ & $\begin{array}{c}\mathrm{V} \\
\mathrm{m}^{3} / \mathrm{uac} \\
\end{array}$ \\
\hline 1 & $0-450$ & \multirow{5}{*}{0.005175} & \multirow{5}{*}{0.00062} & \multirow{5}{*}{0.0018} & \multirow{5}{*}{0.00999} & \multirow{5}{*}{67} & 65 \\
\hline 2 & $450-510$ & & & & & & 88 \\
\hline 3 & $510-630$ & & & & & & 68 \\
\hline 4 & $630-870$ & & & & & & 43 \\
\hline 5 & $870-930$ & & & & & & 76 \\
\hline 6 & $930-990$ & \multirow{3}{*}{0.005} & \multirow{3}{*}{0.00085} & \multirow{3}{*}{0.0023} & \multirow{3}{*}{0.00113} & \multirow{3}{*}{62} & 76 \\
\hline 7 & $990-1050$ & & & & & & 63 \\
\hline 8 & $1050-1100$ & & & & & & 45 \\
\hline 9 & $1100-1290$ & 0.0065 & 0.0009 & 0.00225 & 0.00128 & 62 & 62 \\
\hline 10 & $1290-1425$ & \multirow{2}{*}{0.00675} & \multirow{2}{*}{0.0009} & \multirow{2}{*}{0.00235} & \multirow{2}{*}{0.00132} & \multirow{2}{*}{56} & 62 \\
\hline 11 & $1425-1470$ & & & & & & 38 \\
\hline 12 & $1470-1590$ & \multirow{5}{*}{0.005} & \multirow{5}{*}{0.001} & \multirow{5}{*}{0.0023} & \multirow{5}{*}{0.00116} & \multirow{5}{*}{51} & 38 \\
\hline 13 & $1590-1710$ & & & & & & 50 \\
\hline 14 & $1710-1830$ & & & & & & 40 \\
\hline 15 & $1830-1890$ & & & & & & 78 \\
\hline 16 & 1890 & & & & & & 68 \\
\hline
\end{tabular}

\section{Обсуждение результатов}

Для расчета задается количество этапов, отличающихся исходными данными, для каждого этапа задается время начала и конца этапа, производительность и состав осветленной воды. Полученное в результате расчета каждого этапа распределение всех компонентов является исходным для расчета следующего этапа. Проводится расчет следующего этапа с новыми исходными данными в соответствии с табл. 1 .

По программе «Createscheme» проведено 3 цикла расчетов, отличающихся заданием исходных данных (таблица 1):

1- пять этапов с изменяющимся составом осветленной воды, для каждого этапа усредненная скорость; 
2 -тринадцать этапов с изменяющейся скоростью потока, для каждого этапа усредненный состав осветленной воды;

3- шестнадцать этапов с переменной скоростью и переменным составом осветленной воды.

На рис. 2 и 3 представлены расчетные выходные кривые на середине слоя и на выходе, полученные по программе при учете изменения граничных условий (производительности и состава очищаемой воды).

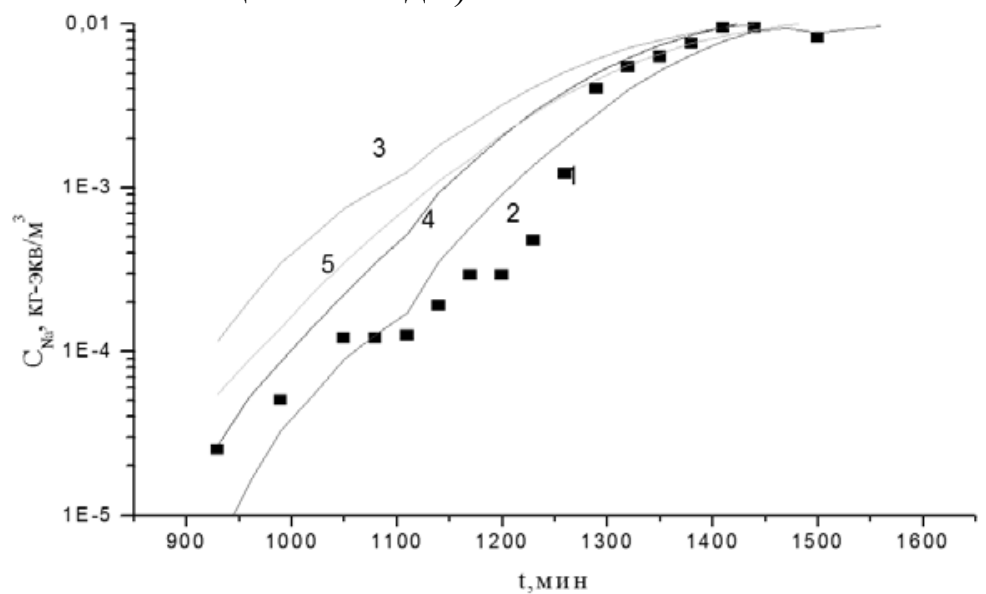

Рис. 2. Выходные кривые натрия в среднем сечении фильтра: 1-эксперимент, 2-расчет с учетом изменения состава воды и производительности, 3-расчет с учетом изменения производительности, 4-расчет с учетом изменения состава воды, 5- расчет

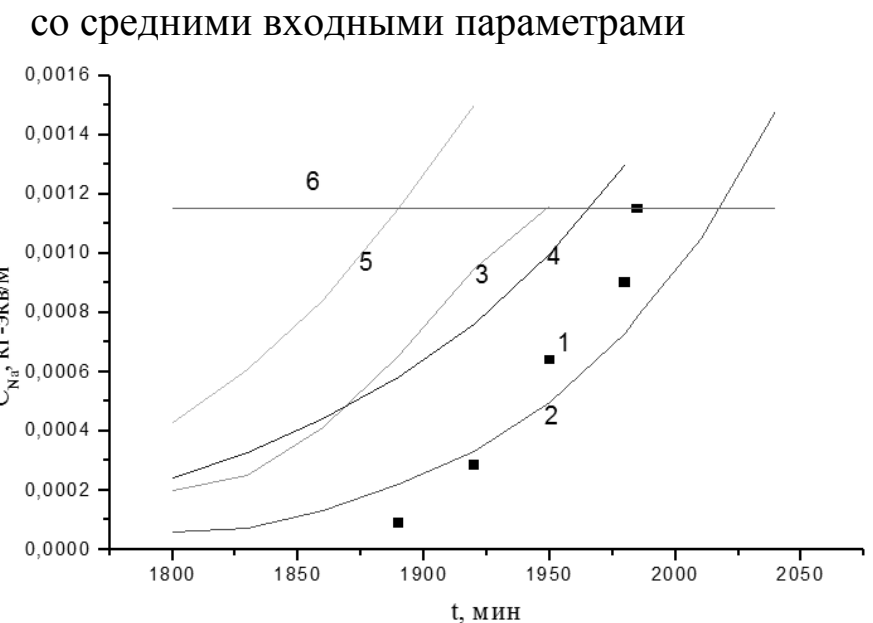

Рис. 3. Выходные кривые натрия на выходе фильтра: 1-эксперимент, 2-расчет с учетом изменения состава воды и производительности, 3-расчет с учетом изменения производительности, 4-расчет с учетом изменения состава воды, 5- расчет с средними входными параметрами, 6-заданная концентрация натрия в момент окончания фильтроцикла.

Экспериментальные выходные кривые в среднем сечении фильтра являлись основой для сопоставления с кривыми, рассчитанными по математической модели с изменением входных параметров (рис.2).

Выходные кривые на выходе фильтра (рис.3) получены только до проскока натрия, так как фильтр во время экспериментов был включен в общую технологическую схему.

Проведенные расчеты показали, что экспериментальные выходные кривые удовлетворительно совпадают с расчетными при учете изменения скорости потока и 
состава осветленной воды в течении рабочего цикла. На рис.4 представлено распределение натрия по слою катионита в моменты изменения концентрации компонентов на входе в фильтр (табл.1). Как следует из этих данных, увеличение концентрации натрия и общего солесодержания входного раствора приводит к большому расширению зоны натрия в слое (этапы 8-9-10) по сравнению с уменьшением концентрации на входе (этапы 10-11-12).

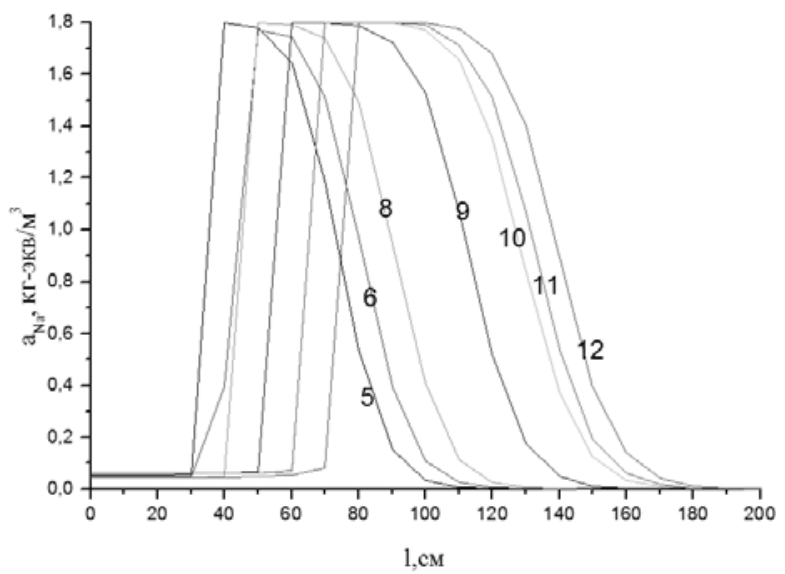

Рис. 4. Распределение иона натрия по слою катионита в моменты изменения концентраций компонентов в очищаемой воде на входе в фильтр

На рис.5 представлено распределение по слою всех компонентов в момент проскока натрия, рассчитанное по программе «Createscheme», которое было исходным для расчета последующих стадий процесса очистки осветленной воды на катионите с учетом изменения входных параметров.

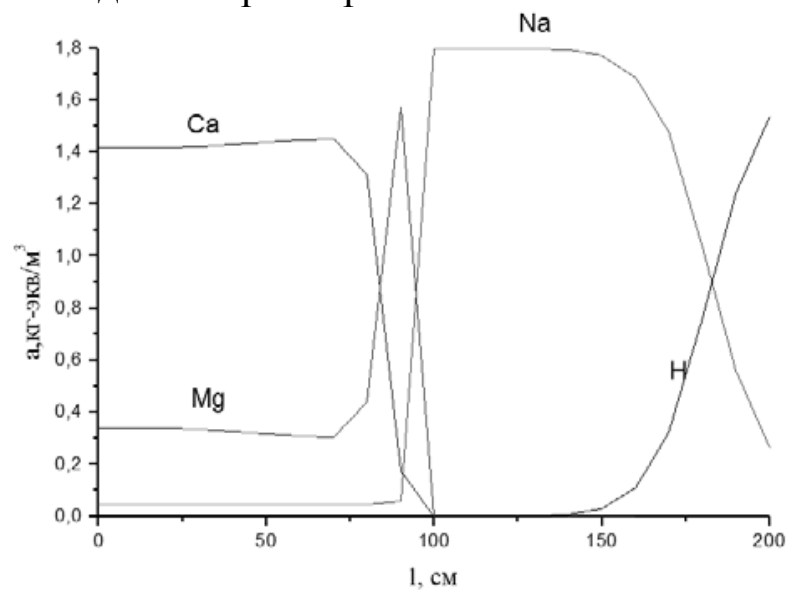

Рис. 5. Расчетное распределение катионов по слою катионита в момент проскока натрия

\section{Заключение}

Результаты проведенного промышленного эксперимента на катионите КУ-2 в водородной форме доказали адекватность математической модели многокомпонентной динамики ионного обмена, изложенной в сообщении 1 , реальному процессу очистки воды в промышленном фильтре.

Учет изменения входных параметров дает возможность предсказывать результаты работы фильтра при изменении исходных данных, на основе чего - менять 
режим его работы в соответствии с рассчитанными данными при изменившихся условиях

\section{Список литературы}

1. Комарова И.В., Закова И.М., Одинцов В.Ю., Лебедев В.Ю. Теплоэнергетика. 1983. № 7. C. 13-16

2. Прудковский А.Г., Комарова И.В., Галкина Н.К. Сорбционные и хроматографические прочессы. 2014. Т. 14. № 5. С.824-831

\section{References}

1. Komarova I.V., Zakova I.M., Odincov V.Ju., Lebedev V.Ju., Teplojenergetika, 1983, No 7, pp. 13-16.

2. Prudkovskii A.G., Komarova I.V., Galkina N.K., Sorbtsionnye $i$ khromatograficheskie protsessy, 2014, Vol. 14, No 5, pp.824-831.

Комарова Ирина Владимировна к.х.н., старший научный сотрудник, лаборатория сорбционных методов, ГЕОХИ РАН

Komarova Irina V. - Ph.D. (Chem.), Senior researcher, Lab of Sorption Methods, GEOKHI RAS

Галкина Надежда Константиновна - к.Х.н., старший научный сотрудник, лаборатория сорбционных методов, ГЕОХИ РАН

Прудковский Андрей Гаральдович - д.ф-м.н., старший научный сотрудник, лаборатория сорбционныХ методов, ГЕОХИ РАН,

Galkina Nadezda K. - Ph.D. (Chem.), Senior researcher, Lab. of Sorption Methods, GEOKHI RAS

Prudkovskii Andrei G. - Dr.Sci. (Phys/Math.), Leading researcher, Lab of Sorption Methods, GEOKHI RAS 